\title{
Recent Advances in the Application of Digital Holographic Microscopy
}

\author{
E.M. MihAYLOVA* \\ Agricultural University, Plovdiv, Bulgaria
}

\begin{abstract}
Holography is a technique by which a wavefront can be recorded and subsequently reconstructed in the absence of the original wavefront. In conventional holography, invented by Gabor, the holograms are photographically recorded and optically reconstructed. Digital holography is an emerging new field in imaging applications, mainly in microscopy. By replacing the photochemical procedures with electronic recording of a hologram and its digital reconstruction, a wide range of new imaging capabilities become available. Once the amplitude and the phase of the light wave are recorded numerically, one can easily subject these data to a variety of manipulations, and so digital holography offers capabilities not available in conventional holography. An increasing number of researchers are realizing and exploiting the remarkable capabilities of digital holography. A brief review of the research carried out at the Agricultural University of Plovdiv in the area of digital holographic microscopy is presented. Recent applications of DIHM in materials studies are reported, in particular studies of polymer-zeolite nanocomposites.
\end{abstract}

DOI: 10.12693/APhysPolA.135.1129

PACS/topics: digital holographic microscopy, zeolite nanocomposites

\section{Introduction}

In conventional holography, invented by Gabor [1] the holograms are photographically recorded and optically reconstructed. Digital holography refers to the acquisition and processing of holograms with a digital sensor array [2] such as a charge-coupled device (CCD). In digital holography the reconstruction process is implemented by multiplication of the digital hologram by the numerical description of the reference wave, and convolution of the result with the impulse response function of the optical system. Quantitative information about the intensity and the phase distribution of the numerically reconstructed wave field can be obtained from the digital holograms. Fast imaging is possible, limited only by video acquisition rate.

Holographic microscopy was first proposed by Gabor [1]. It offers high-contrast 3-D images of microscopic samples in depth. Digital holographic microscopy (DHM) is a very advanced imaging technology because a single digital hologram contains the information necessary for the microscopic object volume reconstruction $[3,4]$. Numerical reconstruction of the digital holograms provides an efficient method of virtual focusing throughout the depth of a microscopic sample, which is a unique feature of the DHM.

The recent research developments of digital holographic microscopy at the Agricultural University of Plovdiv belongs to two fields: applications of DHM in life sciences $[5,6]$ and applications of DHM in materials studies $[5,7-9]$.

*e-mail: emmihaylova@gmail.com

\section{Experimental procedure}

\subsection{Optical setup of the digital holographic microscope}

Digital in-line holographic microscope (DIHM) was developed at the Agricultural University of Plovdiv [10]. The light source is a diode laser (Lasiris) with a wavelength of $673.2 \mathrm{~nm}$ and output of $7 \mathrm{~mW}$. The laser radiation is focused onto a pinhole after which the intensity is controlled by a polarizer (Fig. 1). After the pinhole, the spherical wave passes through the object: the diffracted by the object and the nondiffracted wave interfere as a hologram, which is recorded on a CCD sensor. The intensity and the phase are reconstructed numerically [11].

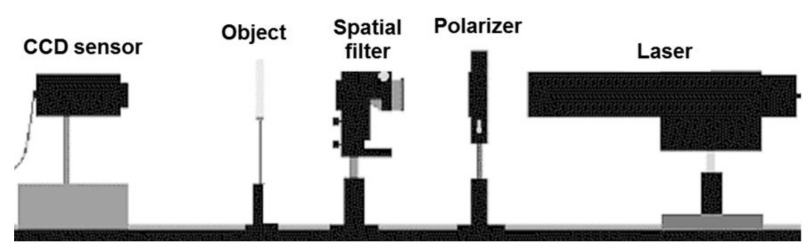

Fig. 1. Optical set-up of the digital in-line holographic microscope.

\subsection{Polymer-zeolite nanocomposite samples}

Zeolite doped polymer nanocomposites are extensively studied advanced materials. A recently reported diacetone-acrylamide-based (DA-based) low-toxicity photopolymer [12] is an attractive novel material for holographic applications. For many applications it is desirable to modify the holographic photopolymers in order to reduce the shrinkage. It has been shown that addition of zeolite nanoparticles to acrylamide-based photopolymers can reduce undesired shrinkage during holographic recording [13]. The effect of zeolite nanoparticles with 
BEA type framework structure on the optical properties of a diacetone-acrylamide (DA)-based holographic photopolymer has been previously investigated [14]. It is shown that doping of the DA photopolymer with BEA zeolite nanoparticles results in a significant increase in refractive index modulation due to holographic photopolymer being a host material for the porous BEA nanoparticles. This is due to the large size of the DA monomer molecules, which restricts monomer migration into the zeolite pores and thus the pores remain empty, making them suitable for application in holographic sensors [14].

The DA photopolymer solution was prepared by adding $1.0 \mathrm{~g}$ DA, $0.2 \mathrm{~g}$ N,N-methylene-bisacrylamide, $2 \mathrm{ml}$ triethanolamine and $4 \mathrm{ml}$ Erythrosin B $0.11 \mathrm{wt} / \mathrm{vol} \%$ dye solution to $10 \mathrm{ml}$ of $20 \mathrm{wt} / \mathrm{vol} \%$ polyvinyl alcohol. $80 \mu \mathrm{m}$ photopolymer layers doped with $0.5 \mathrm{wt} \% \mathrm{Ag}-$ BEA nanoparticles were prepared using gravity settling techniques. A two-beam holographic optical setup was used to record un-slanted transmission gratings using a $532 \mathrm{~nm}$ diode laser. Diffraction gratings were recorded in the nanocomposite layers at a spatial frequency of $130 \pm 10 \mathrm{l} / \mathrm{mm}$. The total intensity in both beams was $1 \mathrm{~mW} / \mathrm{cm}^{2}$, and the exposure energy for recording was $50 \mathrm{~mJ} / \mathrm{cm}^{2}$.

\section{Results and discussion}

Digital in-line holographic microscopy was employed to study the structure of diacetone-based photopolymer layers doped with AgBEA zeolite nanoparticles. All photopolymer samples were of equal thickness of $50 \mu \mathrm{m}$.

Figure 2 shows the experimental results for a diffraction grating with spatial frequency of $70 \mathrm{l} / \mathrm{mm}$ recorded in DA-based photopolymer doped with $0.5 \mathrm{wt} \% \mathrm{AgBEA}$ zeolite nanoparticles. The reconstructed images show that the DA-based photopolymer doped with AgBEA zeolite nanoparticles, before recording of a diffraction grating, has clustered structure. The clusters are cylindrical and are aligned in rows divided by empty spaces ("pores"). The clusters and the pores are typically $15 \mu \mathrm{m}$ wide and have variable length.

Figure 3 shows the experimental results for a diffraction grating with spatial frequency of $100 \mathrm{l} / \mathrm{mm}$ recorded in DA-based photopolymer doped with $0.5 \mathrm{wt} \% \mathrm{Ag}$ BEA zeolite nanoparticles. The hologram reconstructions clearly show that the AgBEA zeolite nanoparticles are concentrated in clusters aligned in rows having the same spatial frequency as the diffraction grating.
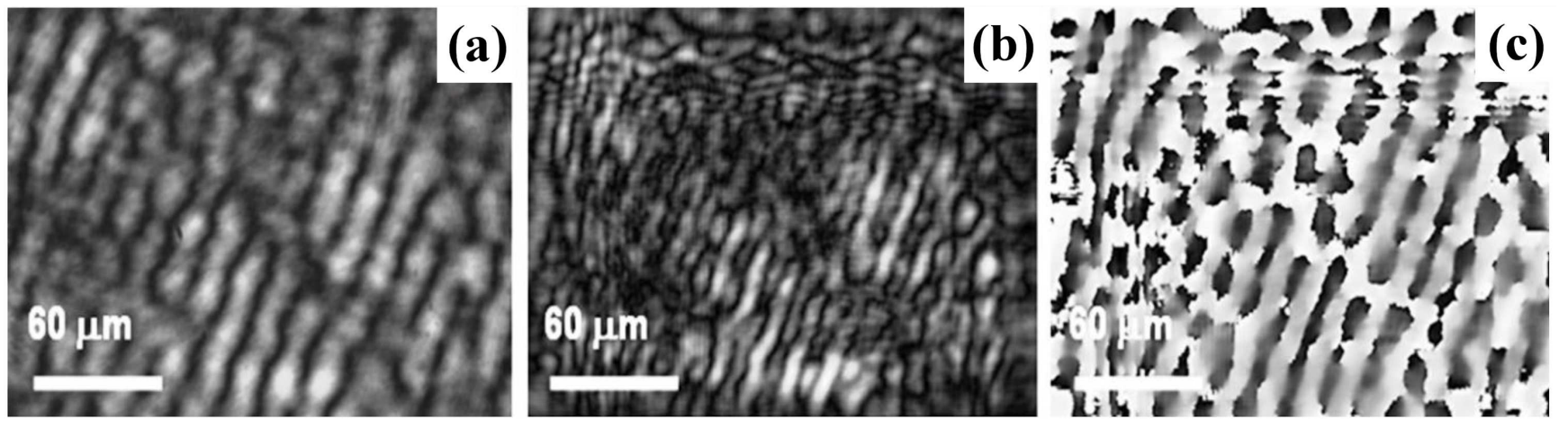

Fig. 2. Images of diffraction grating with spatial frequency of $70 \mathrm{l} / \mathrm{mm}$ recorded in DA-based photopolymer doped with 0.5 wt\% AgBEA zeolite nanoparticles: (a) digital hologram, (b) reconstructed intensity, (c) reconstructed phase.
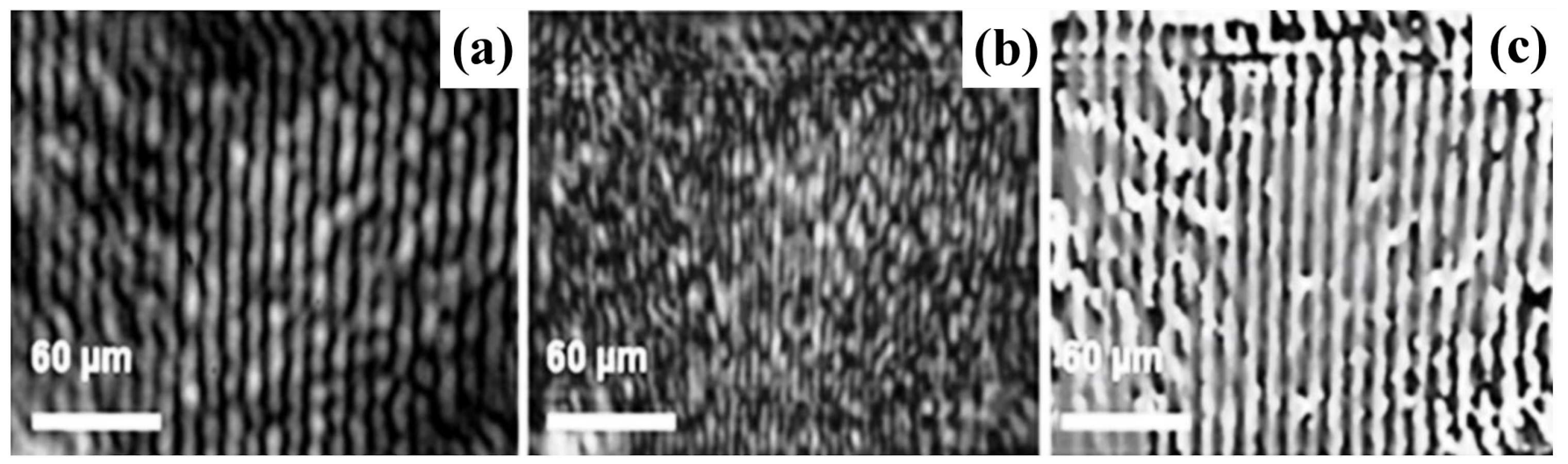

Fig. 3. Images of diffraction grating with spatial frequency of $100 \mathrm{l} / \mathrm{mm}$ recorded in DA-based photopolymer doped with 0.5 wt\% AgBEA zeolite nanoparticles: (a) digital hologram, (b) reconstructed intensity, (c) reconstructed phase. 
During recording the clusters diameters shrink approximately $10 \%$. This leads to higher concentration of AgBEA zeolite nanoparticles in a unit volume. Therefore doping of the DA photopolymer with AgBEA zeolite nanoparticles results in a significant increase in refractive index modulation, reported previously [14]. It can be concluded that doping of holographic photopolimers with porous zeolite nanoparticles increase the refraction index modulation and thus improve the holographic properties of the host photopolymer.

\section{Conclusion}

It was demonstrated that digital in-line holographic microscopy can be used to study the structure of holographic photopolymers doped with zeolite nanoparticles. The results show that the DA-based photopolymer doped with AgBEA zeolites, before recording of a diffraction grating, has clustered structure. Once a diffraction grating is recorded the clusters become linearly aligned in rows having the same spatial frequency as the diffraction grating. During recording the clusters diameters shrink approximately $10 \%$. This leads to higher concentration of AgBEA zeolite nanoparticles in a unit volume. It can be concluded that doping of holographic photopolymers with porous zeolite nanoparticles increase the refraction index modulation and thus improve the holographic properties of the host photopolymer. Other zeolites with hierarchically porous structures could also be beneficial for the improvement of the properties of holographic photopolymers.

\section{Acknowledgments}

The authors are thankful to Prof. Svetlana Mintova, Laboratoire Catalyse \& Spectrochimie, University of Caen, 14050 Caen Cedex, France for providing the AgBEA zeolite nanoparticles used in the research. The authors are thankful to Dervil Cody, Centre for Industrial and Engineering Optics, Dublin Institute of Technology, Ireland for the preparation of the diacetone-acrylamide (DA)-based holographic photopolymer samples. This research has been supported by the Agricultural University of Plovdiv, Bulgaria.

\section{References}

[1] D. Gabor, Nature 161, 777 (1948).

[2] J.W. Goodman, R.W. Lawrence, Appl. Phys. Lett. 11, 77 (1967).

[3] U. Schnars, W. Juptner, Appl. Opt., 33, 179 (1994).

[4] F. Charriere, N. Pavillon, T. Colomb, C. Depeursinge, T.J. Heger, E.A.D. Mitchell, P. Marquet, B. Rappaz, Opt. Express 14, 7005 (2006).

[5] E. Mihaylova, I. Peruhov, Agrarni Nauki 7, 87 (2015).

[6] I. Peruhov, E. Mihaylova, Applications of holographic microscopy in life sciences, Chapter 13 in: Holography - Basic Principles and Contemporary Applications, Ed. E Mihaylova, Intech, 2013, p. 319.

[7] I. Peruhov, E. Mihaylova, Agrarni Nauki 7, 93 (2015).

[8] I. Peruhov, E. Mihaylova, Ferroelectrics 493, 172 (2016).

[9] I.T. Peruhov, E.M. Mihaylova, Bulgarian Chem. Communicat. 48G, 71 (2016)

[10] I. Peruhov, E. Mihaylova, Topics Chem. Mater. Sci. 6, 73 (2012)

[11] O. Skotheim, V.L. Tuft, Holo Vision 2.2.1, software package for numerical reconstruction and analysis of digitally sampled holograms, Norwegian University of Science and Technology, Norway 2001.

[12] D. Cody, I. Naydenova, E. Mihaylova, J. Opt. 14, 1 (2012)

[13] D. Cody, M. Moothanchery, E. Mihaylova, V. Toal, S. Mintova, I. Naydenova, Adv. Mater. Sci. Engin. 2016, 8020754 (2016).

[14] D. Cody, E. Mihaylova, L. O'Neill, T. Babeva, H. Awala, R. Retoux, S. Mintova, I. Naydenova, $O p$ tic. Mater. 37, 181 (2014) 